fact that a Friedberg numbering $\tau$ does not satisfy the recursion theorem relative to $\tau$ (footnote 2 ) may provide some limitation on the usefulness of replacing Gödel numberings by Friedberg numberings in certain technical investigations.

\title{
BIBLIOGRAPHY
}

1. R. Friedberg, Three theorems on recursive enumeration, J. Symbolic Logic 23 (1958), 309-316.

2. S. C. Kleene, Introduction to metamathematics, Van Nostrand, New York, 1953.

3. H. Rogers, Gödel numberings of partial recursive functions, J. Symbolic Logic 23 (1958), 331-341.

INSTITUTE FOR AdVANCED STUdy

\section{THE CLOSED CONVEX HULL OF CERTAIN EXTREME POINTS}

DWIGHT B. GOODNER

Let $H$ be a Hausdorff space and let $C(H)$ be the space of bounded continuous real-valued functions on $H, C(H)$ having the usual supremum norm. Also, let $S$ be the unit ball of $C(H)$, let $E$ be the set of extreme points of $S$, and let $K$ be the set of characteristic functions of open-and-closed sets in $H$.

It is known $[1, \S 3, \mathrm{p} .10]$ that when $H$ is compact, $S$ is the norm closed convex hull of $E$ if and only if $H$ is totally disconnected. It is known also $[4$, p. 103] that if $H$ is extremally disconnected, then $S$ is the norm closed convex hull of $E$. The purpose of this paper is to give a necessary and sufficient condition for $S$ to be the norm closed convex hull of $E$. The theorem for compact $H$ mentioned above is a special case of our result (cf. [3, p. 247]). We are indebted to the referee for observing that if $H$ is completely regular, our result follows at once from the compact case (cf. [3, p. 88]). Our condition is also necessary and sufficient for each of the subsets $E$ and $K$ to be fundamental (cf. [2, p. 35]) in $C(H)$.

If $f$ is a function in $C(H)$, we shall call the set

$$
Z(f)=\{h: h \in H, f(h)=0\}
$$

the zero-set of the function $f$. We observe that if $r$ is a real number, then the sets $\{h: f(h)=r\},\{h: f(h) \geqq r\}$, and $\{h: f(h) \leqq r\}$ are zero-

Received by the editors February 7, 1963. 
sets of the functions $g$, inf $\{g, 0\}$, and $\sup \{g, 0\}$, respectively, where $g$ is a function in $C(H)$ defined by $g(h)=f(h)-r$ for all $h$ in $H$ and 0 is the zero element of $C(H)$. A subset of $H$ will be called a zero-set in $H$ if and only if it is the zero-set of some function $f$ in $C(H)$ (cf. [3, p. 14]). We shall say that $H$ has the zero-set separation property if and only if for each pair of disjoint zero-sets $Z_{1}$ and $Z_{2}$ in $H$ there exist disjoint open-and-closed subsets $H_{1}$ and $H_{2}$ in $H$ such that $Z_{1} \subset H_{1}, Z_{2} \subset H_{2}$, and $H_{1} \cup H_{2}=H$ (cf. [3, p. 246]).

THEOREM. The unit ball $S$ of $C(H)$ is the norm closed convex hull of its extreme points if and only if $H$ has the zero-set separation property.

Proof. Let $H$ have the zero-set separation property, let $\epsilon$ be a real number greater than zero, let $F$ be any element of $S$, and let $N$ be the smallest integer greater than or equal to $2 / \epsilon$. For each integer $n$, $0 \leqq n \leqq N-1$, let $U_{n}=\{h: F(h) \geqq 1-n \epsilon\}$ and let $L_{n}=\{h: F(h) \leqq 1-n \epsilon\}$. Also, let $U_{N}=\{h: F(h) \geqq-1\}$ and let $L_{N}=\{h: F(h) \leqq-1\}$. We see that $U_{n}$ and $L_{n}$ are zero-sets for $0 \leqq n \leqq N$ and that $U_{n} \cap L_{n+1}=\varnothing$ for $0 \leqq n \leqq N+1$. By hypothesis there exist disjoint open-andclosed sets $P_{n}$ and $Q_{n+1}$ such that $P_{n} \supset U_{n}, Q_{n+1} \supset L_{n+1}$, and $P_{n} \cup Q_{n+1}$ $=H$. Let $Q_{0}=P_{N}=H$. The $\bigcup_{n=0}^{N}\left(P_{n} \cap Q_{n}\right)=H$ and if $x$ is a member of $P_{n} \cap Q_{n}$, then $|F(x)-(1-n \epsilon)|<\epsilon$.

The characteristic function $K_{n}$ of the set $P_{n} \cap Q_{n}$ is continuous since $P_{n} \cap Q_{n}$ is open-and-closed. If $k_{n}= \pm 1$, it is easily verified that $\sum_{n=0}^{N} k_{n} K_{n}$ is an extreme point of $S$. Let $t_{n}=n \epsilon / 2$ for $1 \leqq n<N$ and let $t_{N}=1$. Form the functions

$$
\begin{aligned}
& g_{0}=\sum_{j=0}^{N} K_{j} \\
& g_{1}=\left(1-t_{1}\right) g_{0}+t_{1}\left(g_{0}-2 K_{1}\right)=\sum_{i=0}^{1}(1-i \epsilon) K_{i}+\sum_{j=2}^{N} K_{j}, \\
& g_{n}=\left(1-t_{n}\right) g_{n-1}+t_{n}\left(g_{n-1}-2 K_{n}\right)=\sum_{i=0}^{n}(1-i \epsilon) K_{i}+\sum_{j=n+1}^{N} K_{j}, \\
& g_{N}=\sum_{i=0}^{N-1}(1-i \epsilon) K_{i}-K_{N} .
\end{aligned}
$$

The function $g_{N}$ is a member of the convex hull $E^{\prime}$ of $E$ (cf. [2, p. 10]) and $\left\|F-g_{N}\right\|<\epsilon$. It follows that $F$ is in the closure of $E^{\prime}$ and, hence, that $S$ is the closed convex hull of its extreme points. 
Let $S$ be the closed convex hull of $E$ and let $Z(f)$ and $Z(g)$ be disjoint zero-sets in $H$. The function $F$ defined by the relation $F(h)$ $=|f(h)| /(|f(h)|+|g(h)|)$ for all $h$ in $H$ has the properties $0 \leqq F(h) \leqq 1$ for $h$ in $H, F(h)=0$ for $h$ in $Z(f)$, and $F(h)=1$ for $h$ in $Z(g)$. Since $F$ is in $S$, there is a function $G$ in the convex hull of $E$ such that $\|F-G\|$ $<1 / 2$. Since $G$ assumes only a finite number of distinct values on $H$, the sets $H_{1}=\{h: G(h) \leqq 1 / 2\}$ and $H_{2}=\{h: G(h)>1 / 2\}$ are disjoint open-and-closed sets containing $Z(f)$ and $Z(g)$, respectively. Since $H_{1} \cup H_{2}=H$, we see that $H$ has the zero-set separation property. This completes the proof.

Corollary 1. The set $E$ of extreme points of the unit ball $S$ in $C(H)$ is fundamental in $C(H)$ if and only if $H$ has the zero-set separation property.

Proof. Let $F$ be a nonzero element in $C(H)$ and let $\epsilon>0$ be assigned. If $H$ has the zero-set separation property, by the theorem there is a function $g$ in the convex hull of $E$ such that $\|(F /\|F\|)-g\|$ $<\epsilon /\|F\|$. It follows that $\|F-(\|F\| g)\|<\epsilon$ and, hence, that $E$ is fundamental in $C(H)$.

If the set $E$ of extreme points of $S$ is fundamental in $C(H), H$ can be shown to have the zero-set separation property by an argument similar to an argument used in the proof of the above theorem. We omit the details.

COROLlaRy 2. The set $K$ of all characteristic functions of open-andclosed subsets of $H$ is fundamental in $C(H)$ if and only if $H$ has the zeroset separation property.

Proof. If we note that the previously constructed function $g_{N}$ is a linear combination of elements in $K$, the proof follows easily. The details of the proof are left to the reader.

\section{REFERENCES}

1. W. G. Bade, The space of all continuous functions on a compact Hausdorff space, Univ. of California, Berkeley, 1957, unpublished seminar notes.

2. M. M. Day, Normed linear spaces, Ergebnisse der Mathematik, Academic Press, New York, 1962.

3. L. Gillman and M. Jerison, Rings of continuous functions, Van Nostrand, Princeton, N. J., 1960.

4. D. B. Goodner, Projections in normed linear spaces, Trans. Amer. Math. Soc. 69 (1950), 89-108.

Florida State University 